\title{
EXTREME ULTRAVIOLET EXPLORER OPTICAL IDENTIFICATION CAMPAIGN. III. A SOUTHERN HEMISPHERE SAMPLE OF ACTIVE LATE-TYPE STARS AND WHITE DWARFS
}

\author{
Nahide Craig, ${ }^{1}$ Damian J. Christian, Jean Dupuis, and Bryce A. Roberts \\ Center for EUV Astrophysics, 2150 Kittredge Street, Berkeley, California 94720-5030 \\ Electronic mail: ncraig@cea.berkeley.edu \\ Received 1997 February 27; revised 1997 March 21
}

\begin{abstract}
We present optical identifications of 30 previously unidentified extreme ultraviolet (EUV) and soft x-ray sources coinciding with the position of faint sources detected during the all-sky surveys of the Extreme Ultraviolet Explorer (EUVE) in the 58-174 $\AA$ (0.071-0.214 keV) band and of the ROSAT Position Sensitive Proportional Counter (PSPC) in the 5-120 $\AA$ (0.1-2.4 keV x-ray) band. We present low-resolution spectra of the possible optical counterparts of the x-ray and EUVE sources obtained with 1.5 m telescope at CTIO in 1995 May and December and 1996 February. The optical counterparts for 23 sources are identified as late-type ( $\mathrm{dKe}$ and $\mathrm{dMe}$ ) stars, and 4 are identified as new DA white dwarfs. All spectra of sources identified as late-type stars show the Balmer series and $\mathrm{Ca} \mathrm{H}$ and $\mathrm{K}$ lines in emission, indicating the signature of chromospheric activity. For the white dwarfs, the joint detection by the ROSAT PSPC and the EUVE Lexan band indicates that they have a low-abundance of metals and that their EUV emission is mostly attenuated by the local interstellar medium (ISM). (C) 1997 American Astronomical Society. [S0004-6256(97)00107-6]
\end{abstract}

\section{INTRODUCTION}

The Extreme Ultraviolet Explorer (EUVE) and ROSAT all-sky surveys conducted the first detailed studies in the extreme ultraviolet (EUV) waveband region of the electromagnetic spectrum. The second EUVE source catalog contains 734 objects, 245 of which have no known optical identification ("NOID'; Bowyer et al. 1996). The EUVE source list has been expanded by correlating marginal all-sky survey detections from the Lexan bandpass, centered at $100 \AA$, with ROSAT PSPC detections (Lampton et al. 1997), thus raising the number of EUVE sources to over 900 and raising the NOID number to 310 . The ongoing EUVE Optical Identification Campaign series has reported on a subset of these EUVE sources (cf. Mathioudakis et al. 1995a; Craig et al. 1995; Craig \& Fruscione 1997; Craig 1995, 1996). In this paper we present optical identifications for 23 new late-type (dKe and $\mathrm{dMe}$ ) stars and 4 new DA white dwarfs observed at the Cerro Tololo Inter-American Observatory (CTIO) in 1995 May, 1995 December, and 1996 February, comprising about $20 \%$ of the southern faint NOID list. We present in Sec. 2 a brief description of the source selection criteria from which we derived our source list. In Sec. 3 we describe the optical identification procedure adopted and the derived results. In Sec. 4 we present ground-based optical spectroscopy of objects within the error circle of each EUV source, and based on the results of our analysis we discuss the implications for the EUVE source distribution in Sec. 5.

\footnotetext{
${ }^{1}$ Visiting astronomer, CTIO, National Optical Astronomy Observatories, operated by AURA, Inc., under contract with the National Science Foundation.
}

\section{EUVE OBSERVATIONS}

The EUVE all-sky and deep surveys were primarily conducted over a 6 month period from 1992 July 22 to 1993 January 21. Both surveys are discussed in detail in Bowyer \& Malina (1991) and Bowyer et al. (1996). During the allsky survey, three co-aligned telescopes (the "scanners") scanned the sky over the EUV wavelength range in four bandpasses centered at: Lexan/B (100 $), \mathrm{Al} / \mathrm{Ti} / \mathrm{C}(200 \AA)$, $\mathrm{Ti} / \mathrm{Sb} / \mathrm{Al}(400 \AA)$, and $\mathrm{Sn} / \mathrm{SiO}$ or $(600 \AA)$ ). Simultaneously, a fourth telescope, the Deep Survey (DS) instrument, mapped a $2^{\circ} \times 180^{\circ}$ strip of sky along the ecliptic in the two shortest EUV bandpasses, the DS Lexan/B (67-178 $\AA$ ) and DS Al/C (157-364 $\AA$ ), with an order of magnitude greater sensitivity than the all-sky survey. The effective areas for each telescope and filter combination are given in Bowyer et al. (1996).

Initial EUVE source catalogs, such as the first and the second EUVE source catalogs (Bowyer et al. 1994, 1996), listed source detections at $5 \sigma$ to $5.5 \sigma$ detection threshold levels. The faint EUV source catalog of Lampton et al. (1997) uses the bias search concept, to preselect search locations within a $90^{\prime \prime}$ positional coincidence with ROSAT survey detections, thus allowing a lower EUV detection threshold between $3.4 \sigma$ and $5 \sigma$ to be adopted. This method is sensitive to fainter sources while keeping a tolerable false detection rate.

The present work benefits from the above "biased search" of the EUVE survey, which cross correlates EUVE and ROSAT detections within a 90" positional tolerance (Lampton et al. 1997). Consequently, we detect 166 new EUVE sources, 61 of which are NOIDs. Combining this re- 
TABLE 1. EUVE and ROSAT data for the southern sample of sources.

\begin{tabular}{|c|c|c|c|c|c|c|c|c|c|c|c|c|}
\hline \multicolumn{9}{|c|}{$E U V E$} & \multirow[b]{2}{*}{$\begin{array}{c}\text { Ang. Sep. }{ }^{c} \\
(") \\
(10)\end{array}$} & \multicolumn{3}{|c|}{ ROSAT } \\
\hline $\begin{array}{c}\text { Name } \\
\text { (1) }\end{array}$ & $\begin{array}{c}\text { R.A. } \\
\text { (hh mm ss) } \\
(2)\end{array}$ & $\begin{array}{c}\text { Dec. } \\
\text { (dd mm ss) } \\
\text { (3) }\end{array}$ & $\begin{array}{c}l \\
(4)\end{array}$ & $\begin{array}{c}b \\
(5)\end{array}$ & $\begin{array}{l}T_{\exp } \\
(\mathrm{s}) \\
(6)\end{array}$ & $\begin{array}{c}\text { Count rate } \\
\text { (counts } s^{-1} \text { ) } \\
(7)\end{array}$ & $\begin{array}{c} \pm \sigma \\
\left.(\text { counts s})^{-1}\right) \\
(8)\end{array}$ & $\begin{array}{l}\text { Signif. }^{b} \\
\left(\sigma^{2}\right) \\
(9)\end{array}$ & & $\begin{array}{c}\text { Count rate }^{d} \\
\text { (counts s}{ }^{-1} \text { ) } \\
(11)\end{array}$ & $\begin{array}{c} \pm \sigma \\
\left(\sigma^{2}\right) \\
(12)\end{array}$ & \\
\hline EUVE J0106-637 & 010615 & -634704 & 300.1977 & -53.2651 & 2308 & 0.015 & 0.005 & 13 & $\cdots$ & $\cdots$ & $\cdots$ & $\cdots$ \\
\hline EUVE J0239- 196 & 023915 & -193807 & 201.8320 & -64.2119 & $\cdots$ & $0.003^{\mathrm{e}}$ & $\cdots$ & $\cdots$ & 202 & 0.070 & 0.019 & $1 \mathrm{RXS}$ \\
\hline EUVE J0254-511 & 025430 & -510900 & 267.2802 & -56.5974 & 1985 & 0.023 & 0.006 & 21 & 46 & 0.122 & 0.022 & 1RXS \\
\hline EUVE J0318-363 & 031856 & -362424 & 238.8455 & -57.5253 & 1441 & 0.023 & 0.006 & 12 & 52 & 0.121 & 0.034 & $1 \mathrm{RXS}$ \\
\hline EUVE J0352-492 & 035222 & -491329 & 257.9671 & -48.9938 & $\cdots$ & $0.002^{\mathrm{e}}$ & $\cdots$ & $\cdots$ & $\cdots$ & $\cdots$ & $\cdots$ & $\cdots$ \\
\hline EUVE J0426-379 & 042621 & -375750 & 240.7093 & -44.0768 & 1203 & 0.030 & 0.009 & 23 & 52 & 0.555 & 0.057 & $1 \mathrm{RXS}$ \\
\hline EUVE J0450-086 & 045030 & -083823 & 206.6980 & -30.7675 & 677 & 0.031 & 0.010 & 15 & $\cdots$ & $\cdots$ & $\cdots$ & $\cdots$ \\
\hline EUVE J0609-358 & 060923 & -354935 & 242.5653 & -23.4888 & 1935 & 0.020 & 0.008 & 16 & 57 & 0.367 & 0.024 & 1RXS \\
\hline EUVE J0625-600 & 062555 & -600307 & 269.3013 & -26.3896 & 8493 & 0.008 & 0.002 & 13 & 23 & 0.539 & 0.025 & 1RXS \\
\hline EUVE J0652-596 & 065247 & -594105 & 269.6742 & -23.0164 & 8525 & 0.100 & 0.003 & 19 & 61 & $<0.010$ & $\cdots$ & $1 \mathrm{RXS}$ \\
\hline EUVE J0702-018 & 070256 & -014739 & 215.8403 & 1.7378 & 1030 & 0.030 & 0.009 & 17 & $\cdots$ & $\cdots$ & $\cdots$ & $\cdots$ \\
\hline EUVE J0749-766 & 074907 & -764214 & 288.9152 & -23.1282 & 3476 & 0.038 & 0.006 & 89 & 36 & 0.594 & 0.070 & $1 \mathrm{RXS}$ \\
\hline EUVE J0817-827 & 081802 & -824341 & 295.5179 & -24.2523 & 1912 & 0.020 & 0.006 & 16 & 50 & 0.791 & 0.054 & $1 \mathrm{RXS}$ \\
\hline EUVE J0825-163 & 082552 & -162340 & 238.8073 & 12.3110 & 1221 & 0.027 & 0.008 & 20 & 64 & 0.509 & 0.043 & $1 \mathrm{RXS}$ \\
\hline EUVE J0835-430 & 083537 & -430333 & 261.8855 & -1.4789 & 2156 & 0.026 & 0.008 & 18 & 82 & 0.050 & 0.001 & 1WGA \\
\hline EUVE J0836-449 & 083627 & -445350 & 263.4458 & -2.4634 & 2046 & 0.023 & 0.007 & 18 & 90 & $<0.05$ & $\cdots$ & $1 \mathrm{RXS}$ \\
\hline EUVE J0913-629 & 091339 & -625946 & 280.9109 & -9.8636 & 3616 & 0.013 & 0.004 & 15 & 81 & 0.145 & 0.020 & IRXS \\
\hline EUVE J1118-569 & 111850 & -565847 & 290.5352 & 3.6464 & 2171 & 0.017 & 0.005 & 16 & $\cdots$ & $\cdots$ & $\cdots$ & $\cdots$ \\
\hline EUVE J1131-346 & 113153 & -343750 & 284.8747 & 25.4362 & 1247 & 0.024 & 0.008 & 14 & 92 & 0.656 & 0.076 & $1 \mathrm{RXS}$ \\
\hline EUVE J1145-553 & 114555 & -552036 & 293.6464 & 6.3428 & 2816 & 0.018 & 0.005 & 22 & 47 & 0.456 & 0.043 & $1 \mathrm{RXS}$ \\
\hline EUVE J1254-471 & 125422 & -470536 & 303.4500 & 15.7755 & 816 & 0.031 & 0.010 & 17 & 62 & 0.10 & 0.024 & $1 \mathrm{RXS}$ \\
\hline EUVE J1305-409 & 130526 & -405450 & 305.7808 & 21.8804 & 813 & 0.028 & 0.011 & 13 & 96 & 0.062 & 0.017 & $1 \mathrm{RXS}$ \\
\hline EUVE J1931-215 & 193126 & -213416 & 17.4553 & -18.1407 & 1033 & $<0.024$ & $\cdots$ & $\cdots$ & 75 & 0.169 & 0.026 & $1 \mathrm{RXS}$ \\
\hline \multicolumn{13}{|c|}{ White Dwarfs } \\
\hline EUVE J0335-348 & 033534 & -344828 & 235.6436 & -54.2443 & 1700 & 0.033 & 0.007 & 37 & 102.8 & 0.063 & 0.005 & 1WGA \\
\hline EUVE J0337-419 & 033719 & -415546 & 247.5821 & -53.2091 & 1809 & 0.029 & 0.007 & 31 & 71.4 & 0.145 & 0.034 & $1 \mathrm{RXS}$ \\
\hline EUVE J0653-564 & 065358 & -562522 & 266.3178 & -21.9914 & 5581 & 0.012 & 0.003 & 18 & $\cdots$ & $\cdots$ & $\cdots$ & $\cdots$ \\
\hline EUVE J1535-774 & 153540 & -772430 & 311.5610 & -17.3805 & 875 & 0.067 & 0.013 & 50 & 16.4 & 0.485 & 0.054 & $1 \mathrm{RXS}$ \\
\hline
\end{tabular}

${ }^{\mathrm{a}} E U V E$ count rate in the Lexan/B band $(58-174 \AA$ or $0.075-0.21 \mathrm{keV})$.

${ }^{\mathrm{b}} E U V E$ detection significance.

${ }^{\mathrm{c}}$ Angular separation between the EUVE and the ROSAT source.

${ }^{\mathrm{d}}$ ROSAT PSPC count rate in the $0.1-2.4 \mathrm{keV}$ band or 5-124 $\AA$ (RASSBSC, Voges et al. 1996; WGA, White et al. 1995 in HEASARC WWW site).

${ }^{\mathrm{e}} E U V E$ Right Angle Program source from McDonald et al. 1994.

sult with the second catalog NOIDs brings the total unidentified source number to 310 . While we have concentrated on observing sources from the "faint" sample, we have also observed sources from the second catalog, such as EUVE J0239- 169 and EUVE J0352-492. These sources are detected with the EUVE Right Angle Program (RAP), which takes advantage of serendipitous, longer, pointed observations with the survey telescopes during pointed guest spectroscopic observations (McDonald et al. 1994). Table 1 shows the EUVE all-sky and pointed data for the NOID sources: the EUVE positions, (EUVE) exposure times, Lexan/B (100 $\AA)$ bandpass count rates and count rate errors, and the detection significance are derived from $E U V E$ all-sky survey data. We obtained the ROSAT PSPC count rates, associated errors, detection significance in the ROSAT survey and the ROSAT source identifications, where available, (the limiting count rate in RASSBSC is 0.05 counts $\mathrm{s}^{-1}$ ) from the ROSAT All Sky Survey Bright Source Catalog (RASSBSC; Voges et al. 1997). These quantities, together with the calculated angular separation between the EUVE and ROSAT source positions, are also shown in Table 1.

\section{OPTICAL OBSERVATIONS}

The procedure for identifying EUVE sources with likely optical candidates in databases has been discussed in detail in the preceding optical identification paper (Craig et al. 1995). Previous optical identification campaigns to identify $E U V E$ sources have found that using a 1-2 meter-class telescope with a moderate resolution spectrograph is adequate for identifying optical counterparts. We obtained spectroscopic data for our southern hemisphere sample of unidentified EUVE sources on 1995 May 20-22, December 19-22, and 1996 February $14-18$ with the $1.5 \mathrm{~m}$ telescope at CTIO.

The CTIO instrumental setup used a Cassegrain spectrograph and LORAL $(1200 \times 800)$ CCD detector with a 300 lines $\mathrm{mm}^{-1}$ grating and a 4."5 entrance slit for the 1995 December and 1996 February observations, covering the wavelength region of $3700-6750 \AA$. For the 1995 May observing run we used the CCD detector GEC 10 covering the wavelength region of $3750-6150 \AA$. Both instrument setups give a spectral resolution of $\sim 9 \AA$.

The CTIO data were reduced using standard IRAF software. The spectra were trimmed and the bias-level sub- 
TABLE 2. Optical data and physical parameters.

\begin{tabular}{|c|c|c|c|c|c|c|c|c|c|}
\hline$\underset{(1)}{\text { Name }}$ & $\begin{array}{l}\text { Source }^{\text {a }} \\
\text { No. } \\
\text { (2) }\end{array}$ & $\begin{array}{c}\text { R.A.opt } \\
\text { (hh mm ss) } \\
\text { (3) }\end{array}$ & $\begin{array}{c}\text { Dec.opt } \\
\text { (dd mm ss) } \\
\text { (4) }\end{array}$ & $\begin{array}{l}\text { Observation } \\
\text { Date } \\
(5)\end{array}$ & $\begin{array}{c}\text { Exp. } \\
T \\
(6)\end{array}$ & $\begin{array}{c}E U V E^{b} \\
\text { Sep. } \\
(7)\end{array}$ & $\begin{array}{c}\text { ROSAT } \\
\text { Sep. } \\
(8)\end{array}$ & $\begin{array}{c}\operatorname{Mag}^{\mathrm{d}} \\
\left(m_{v}\right) \\
(9)\end{array}$ & $\begin{array}{c}\text { Spectral } \\
\text { Type } \\
(10)\end{array}$ \\
\hline EUVE J0106- 637 & $1^{*}$ & 010622 & -634640 & $09 \mathrm{Dec} 95$ & 1200 & 53.4 & 25.9 & 13.8 & $\mathrm{dM} 4 \mathrm{e}$ \\
\hline \multirow[t]{2}{*}{ EUVE J0239- 196} & $1^{*}$ & 023901 & -193703 & $09 \operatorname{Dec} 95$ & 1200 & 38.6 & $\cdots$ & 12.8 & $\mathrm{dM} 0 \mathrm{e}$ \\
\hline & 2 & 023901 & -193648 & $09 \operatorname{Dec} 95$ & 1800 & 22.8 & $\cdots$ & 12.8 & dK5 \\
\hline \multirow[t]{2}{*}{ EUVE J0254-511 } & $1^{*}$ & 025434 & -510831 & 20 Feb 96 & 1200 & 36.0 & 11.0 & 12.7 & $\mathrm{dM} 3 \mathrm{e}$ \\
\hline & 3 & 025436 & -510819 & 20 Feb 96 & 1200 & 63.6 & 19.7 & 15.1 & $\mathrm{dK} 0$ \\
\hline EUVE J0318-363 & $1^{*}$ & 031858 & -362332 & 09 Dec 95 & 900 & 56.9 & 5.0 & 17.2 & $\mathrm{dM} 4 \mathrm{e}$ \\
\hline \multirow[t]{3}{*}{ EUVE J0335-348 } & $2^{*}$ & 033534 & -345000 & 22 Feb 96 & 900 & 92.3 & 44.4 & 16.4 & $\mathrm{DA}$ \\
\hline & 1 & 033528 & -344930 & 22 Feb 96 & 900 & 89.9 & 35.2 & 16.6 & G9 \\
\hline & 4 & 033534 & -345037 & 22 Feb 96 & 300 & 129.6 & 62.1 & 14.5 & G9 \\
\hline EUVE J0337-419 & $1^{*}$ & 033715 & -415524 & 21 Feb 96 & 1800 & 54.9 & 27.8 & 17.0 & DA \\
\hline \multirow[t]{2}{*}{ EUVE J0352-492 } & $1^{*}$ & 035241 & -491428 & 10 Dec 95 & 1800 & 41.5 & $\cdots$ & 13.7 & $\mathrm{dK} 5 \mathrm{e}$ \\
\hline & 2 & 035245 & -491359 & $10 \operatorname{Dec} 95$ & 2700 & 48.6 & $\cdots$ & 14.5 & dKo \\
\hline EUVE J0426-379 & $1^{*}$ & 042617 & -375730 & 10 Dec 95 & 900 & 53.0 & 2.0 & 11.5 & $\mathrm{dK} 4 \mathrm{e}$ \\
\hline \multirow[t]{2}{*}{ EUVE J0450-086 } & $1^{*}$ & 045030 & -083710 & 23 Feb 96 & 180 & 73.8 & 15.8 & 13.2 & $\mathrm{dK} 4 \mathrm{e}$ \\
\hline & 2 & 045029 & -083604 & 23 Feb 96 & 500 & 139.9 & 50.6 & 16.1 & $\mathrm{dM} 0$ \\
\hline EUVE J0609-358 & $1^{*}$ & 060919 & -354929 & 09 Dec 95 & 600 & 56.7 & 1.0 & 11.5 & $\mathrm{dM} 4 \mathrm{e}$ \\
\hline \multirow[t]{2}{*}{ EUVE J0625-600 } & $1^{*}$ & 062556 & -600327 & 09 Dec 95 & 600 & 18.7 & 3.9 & 12.7 & dM5e \\
\hline & 2 & 062555 & -600329 & 09 Dec 95 & 900 & 25.2 & 15.0 & 16.5 & M2 \\
\hline \multirow[t]{2}{*}{ EUVE J0652-596 } & $1^{*}$ & 065252 & -593956 & $21 \mathrm{Feb} 96$ & 1800 & 79.1 & 24.1 & $\cdots$ & $\mathrm{dM} 3 \mathrm{e}$ \\
\hline & 2 & 065249 & -593956 & $21 \mathrm{Feb} 96$ & 1000 & 31.9 & 27.6 & $\cdots$ & $\mathrm{dK} 7$ \\
\hline \multirow[t]{3}{*}{ EUVE J0653-564 } & $3^{*}$ & 065348 & -562449 & 23 Feb 96 & 2700 & 89.8 & 42.8 & 17.0 & $\mathrm{DA}$ \\
\hline & 1 & 065352 & -562548 & $12 \operatorname{Dec} 95$ & 90 & 60.1 & 67.3 & 9.6 & $\mathrm{dK} 7$ \\
\hline & 2 & 06.5355 & -562447 & 22 Feb 96 & 2700 & 42.4 & 17.8 & 14.9 & dG7 \\
\hline \multirow[t]{3}{*}{ EUVE J0702-018 } & 1 & 070257 & -014833 & $23 \mathrm{Feb} 96$ & 900 & 54.0 & 21.2 & 14.2 & $\mathrm{dF} 6$ \\
\hline & 3 & 070256 & -014734 & $23 \mathrm{Feb} 96$ & 600 & 7.2 & 46.4 & 15.1 & $\mathrm{~A} 2$ \\
\hline & $5^{*}$ & 070259 & -014839 & $23 \mathrm{Feb} 96$ & 600 & 63.9 & 24.9 & 14.9 & $\mathrm{dM} 2 \mathrm{e}$ \\
\hline EUVE J0749-766 & $1^{*}$ & 074902 & -764206 & $09 \operatorname{Dec} 95$ & 600 & $\cdots$ & $\cdots$ & 11.8 & dM5e \\
\hline \multirow{2}{*}{ EUVE J0817-827 } & $1^{*}$ & 081740 & -824332 & $09 \operatorname{Dec} 95$ & 300 & 46.8 & 13.1 & $\cdots$ & $\mathrm{dM} 5 \mathrm{e}$ \\
\hline & 2 & 081739 & $\begin{array}{lll}-82 & 43 & 13\end{array}$ & $09 \operatorname{Dec} 95$ & 1000 & 54.4 & 6.2 & $\cdots$ & $\mathrm{dK} 0$ \\
\hline \multirow[t]{3}{*}{ EUVE J0825-163 } & $1^{*}$ & 082552 & -162246 & 21 Feb 96 & 180 & 54.0 & 10.6 & 10.8 & $\mathrm{dM} 0 \mathrm{e}$ \\
\hline & 2 & 082552 & -162308 & $21 \mathrm{Feb} 96$ & 600 & 32.0 & 32.2 & 18.0 & G1 \\
\hline & 3 & 082548 & -162240 & $21 \mathrm{Feb} 96$ & 600 & 79.2 & 51.2 & 14.6 & $\mathrm{dK} 5$ \\
\hline \multirow[t]{4}{*}{ EUVE J0835-430 } & $1^{*}$ & 083539 & -430245 & 23 Feb 96 & 500 & 52.7 & 30.1 & 18.4 & M1Ie \\
\hline & 2 & 083539 & -430139 & $23 \mathrm{Feb} 96$ & 600 & 115.1 & 33.6 & 17.8 & M2I \\
\hline & 3 & 083541 & -430143 & 23 Feb 96 & 600 & 117.3 & 39.9 & 14.9 & $\mathrm{~K} 5$ \\
\hline & 4 & 083544 & -430146 & $23 \mathrm{Feb} 96$ & 600 & 124.8 & 57.5 & 14.5 & A5 \\
\hline EUVE J0836- 449 & $1^{*}$ & 083632 & -445507 & 22 Feb 96 & 1200 & 90.0 & 11.7 & 18.2 & $\mathrm{dM} 1 \mathrm{e}$ \\
\hline & 2 & 083630 & -445527 & 23 Feb 96 & 1200 & 148.8 & 61.2 & $\cdots$ & G9 \\
\hline & 3 & 083633 & -445533 & 23 Feb 96 & 1200 & 148.8 & 61.2 & $\cdots$ & G9 \\
\hline & 4 & 083633 & -445509 & 23 Feb 96 & 1200 & 148.8 & 61.2 & $\cdots$ & G6 \\
\hline EUVE J0913-629 & $1^{*}$ & 91330 & -625909 & 20 Feb 96 & 400 & 71.6 & 12.8 & 11.1 & $\mathrm{~K} 0 \mathrm{e}^{\mathrm{e}}$ \\
\hline & 2 & 91326 & -625925 & 20 Feb 96 & 600 & 92.9 & 32.2 & 16.0 & F8 \\
\hline & 3 & 91335 & -625931 & 20 Feb 96 & 600 & 32.5 & 52.0 & 15.0 & F6 \\
\hline & 4 & 91334 & -625829 & 20 Feb 96 & 900 & 84.6 & 48.4 & 15.9 & K0 \\
\hline EUVE J1118-569 & $1^{*}$ & 111853 & -565748 & 21 Feb 96 & 450 & 61.8 & 10.1 & 14.5 & $\mathrm{dM} 3 \mathrm{e}$ \\
\hline & 2 & 111856 & -565715 & 21 Feb 96 & 450 & 101.1 & 42.4 & 12.9 & $\mathrm{dK} 5$ \\
\hline & 3 & 111847 & -565654 & 21 Feb 96 & 450 & 119.5 & 63.8 & 15.7 & $\mathrm{dK} 0$ \\
\hline EUVE J1131-346 & $1^{*}$ & 113155 & -343626 & 21 Feb 96 & 150 & 86.6 & 12.5 & 11.8 & $\mathrm{dM} 2 \mathrm{e}$ \\
\hline EUVE J1145-553 & $1^{*}$ & 114552 & -552044 & $12 \operatorname{Dec} 95$ & 90 & 32.9 & 15.1 & 10.7 & $\mathrm{dK} 5 \mathrm{e}$ \\
\hline & $2^{*}$ & 114548 & -552029 & $12 \operatorname{Dec} 95$ & 600 & 67.0 & 23.6 & 13.6 & $\mathrm{dM} 3 \mathrm{e}$ \\
\hline EUVE J1254- 471 & 1 & 125422 & -470640 & 21 Feb 96 & 600 & 63.6 & 20.8 & 15.5 & $\mathrm{dK} 5$ \\
\hline & $2^{*}$ & 125423 & -470618 & $21 \mathrm{Feb} 96$ & 300 & 41.6 & 27.2 & 14.9 & $\mathrm{dM} 3 \mathrm{e}$ \\
\hline & 3 & 125426 & -470645 & $21 \mathrm{Feb} 96$ & 450 & 73.8 & 13.6 & 17.2 & dK0 \\
\hline EUVE J1305-409 & $1^{*}$ & 130530 & -405625 & $21 \mathrm{Feb} 96$ & 900 & 103.4 & 7.3 & 13.3 & $\mathrm{dMle}$ \\
\hline & 3 & 130531 & -405604 & 21 Feb 96 & 600 & 86.3 & 15.2 & 15.3 & $\mathrm{dK} 5$ \\
\hline & 4 & 130533 & -405638 & 21 Feb 96 & 180 & 128.6 & 36.6 & 13.1 & G9 \\
\hline & 5 & 130534 & -405622 & $21 \mathrm{Feb} 96$ & 180 & 123.7 & 44.0 & 14.1 & F8 \\
\hline EUVE J1535-774 & $1^{*}$ & 153545 & -772442 & 22 Feb 96 & 600 & 19.5 & 5.7 & 17.7 & DA \\
\hline & 2 & 153539 & -772438 & 22 Feb 96 & 600 & 7.0 & 21.4 & 18.4 & K5 \\
\hline & 3 & 153540 & -772453 & 22 Feb 96 & 600 & 11.4 & 22.8 & 17.3 & K6 \\
\hline & $4 \mathrm{~A}$ & 153548 & -772525 & 22 Feb 96 & 600 & 51.5 & 50.8 & 14.2 & $\mathrm{dK} 5$ \\
\hline & $4 B$ & 153549 & -772525 & 22 Feb 96 & 600 & 51.5 & 50.8 & 14.1 & dM0 \\
\hline & 5 & 153551 & -772354 & 22 Feb 96 & 600 & 59.2 & 46.5 & 16.5 & $\mathrm{dG} 2$ \\
\hline EUVE J1931-215 & $1^{*}$ & 193126 & -213416 & 22 May 95 & 300 & $\cdots$ & $\cdots$ & 12.3 & dM5e \\
\hline
\end{tabular}

${ }^{a}$ Corresponds to the number in the finding chart.

${ }^{b}$ Angular separation between the optical and the EUVE source.

${ }^{c}$ Angular separation between the optical and the ROSAT source
${ }^{d}$ From $V$ bandpass spectrophotometry; the estimated error is $0.5 \mathrm{mag}$

${ }^{e}$ Shows very weak emission. Not included in Table 3.

${ }^{f}$ Optical counterpart. 

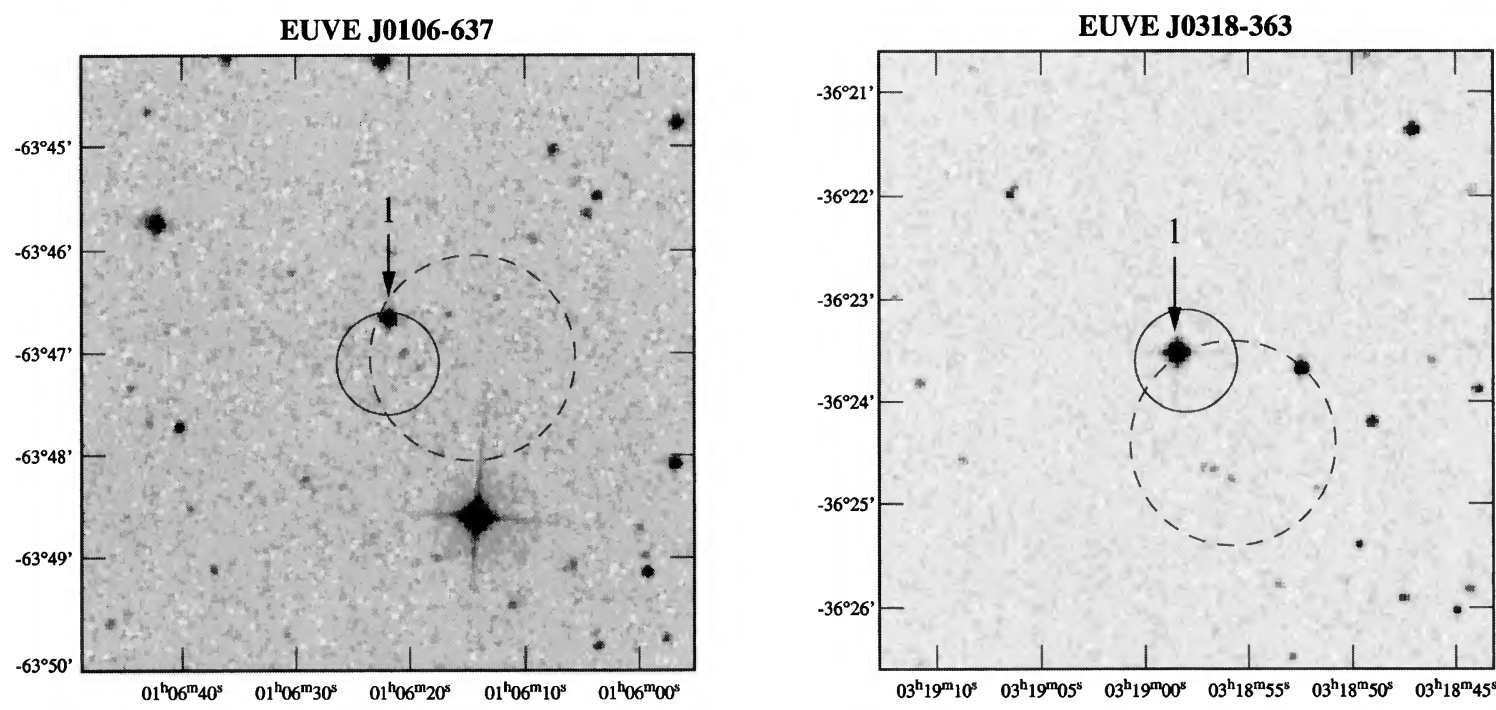

EUVE J0239-196
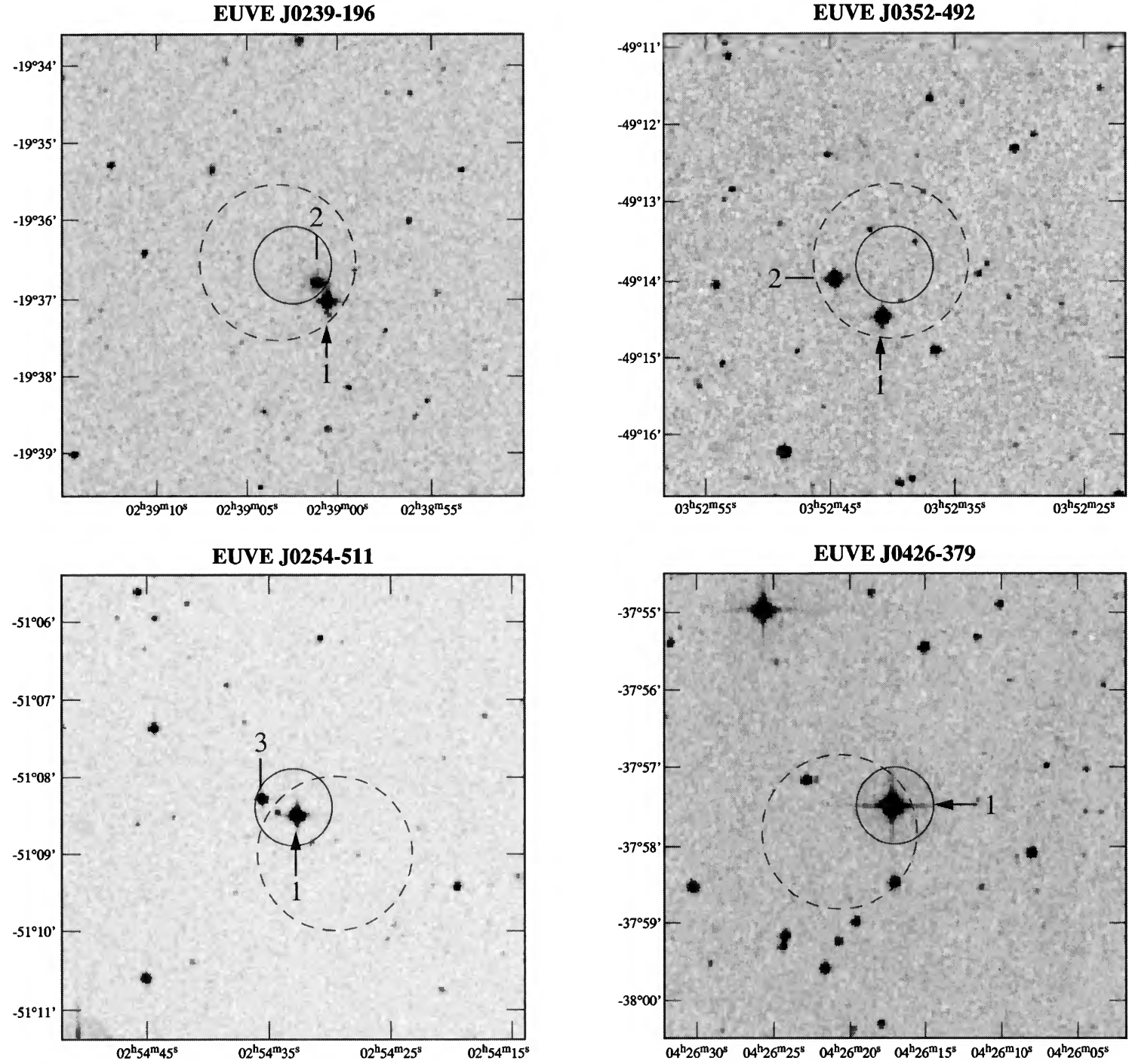

FIG. 1. Finding charts of optical counterparts labeled with the EUVE source name. The candidate marked with arrow is the optical counterpart of the EUVE source as they are are marked with $(*)$ in column 2 of Table 2 .

tracted. Pixel-to-pixel gain variations were removed by applying flat-field correction using an internal quartz lamp, and one-dimensional spectra were extracted. We used an $\mathrm{He}-\mathrm{Ar}$ lamp for wavelength calibration; the resulting rms wave- length errors were approximately $0.1 \AA$. The standard stars EG 274 and LTT 2415 were observed for flux calibration in 1996 February, Feige 110 and LTT 3128 for 1995 December, and LTT 3864 for 1995 May. 

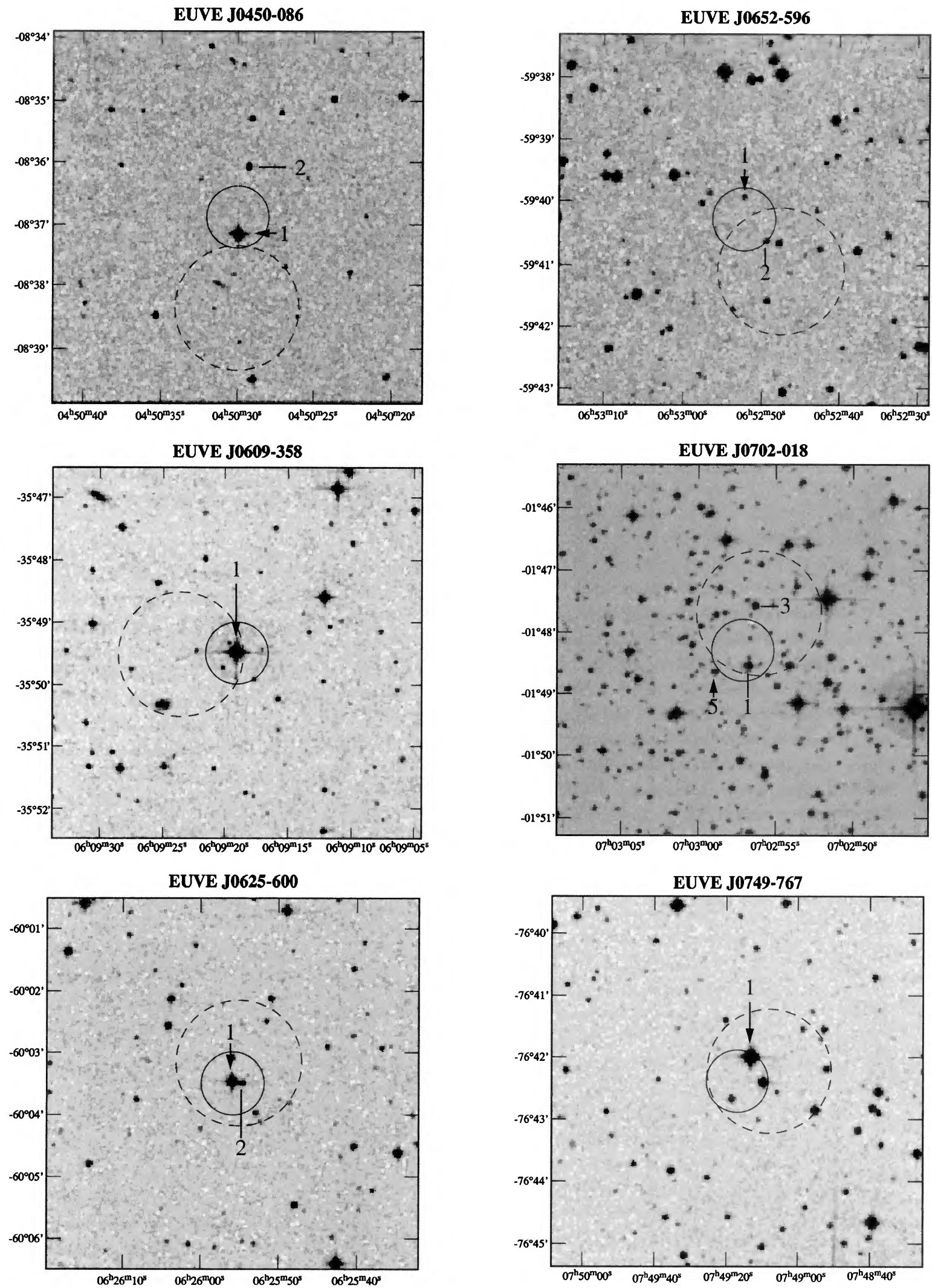

FIG. 1. (continued)

In the CTIO 1995 May run, the wavelength coverage simultaneously excluded $\mathrm{Ca}$ II $\mathrm{H}$ and $\mathrm{K}$ and $\mathrm{H} \alpha$ range. We also did not have the added advantage of using the ROSAT coordinates, which have a pointing accuracy of $30^{\prime \prime}$. The combined effect of these circumstances yielded too few identifications in this observing run.

In the 1995 December and 1996 February observing runs, we used mainly the joint detection source sample. We also

\section{(C) American Astronomical Society - Provided by the NASA Astrophysics Data System}



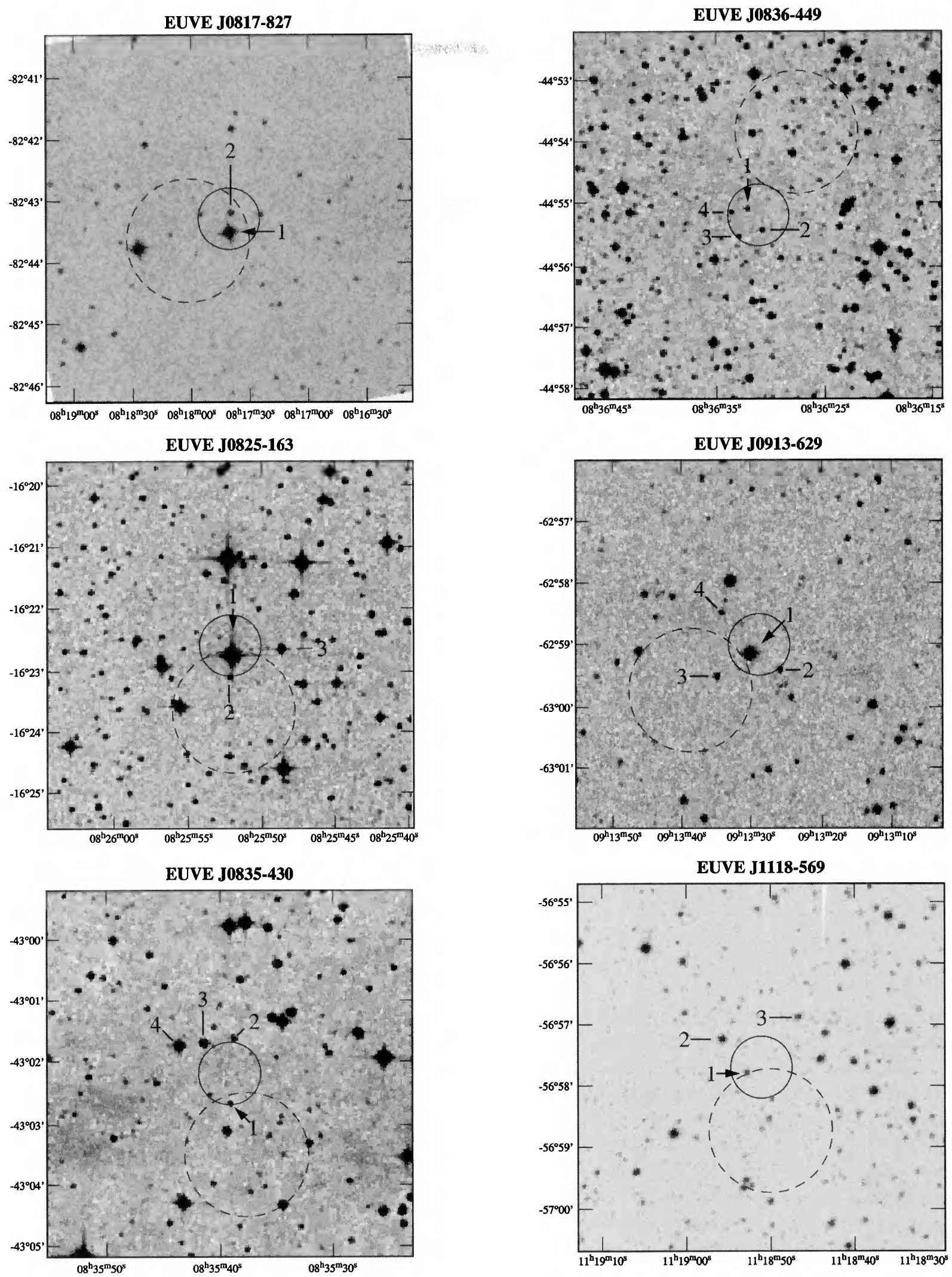

FIG. 1. (continued)

had a much higher rate of successful identifications using a new LORAL CCD chip with a wavelength coverage of $3700-6750 \AA$ and tighter constrains for ROSAT source coordinates. We have observed 77 fields during these three observing runs. We list identifications of 24 late-type stars and 4 white dwarfs as possible EUVE optical counterparts. The optical identification of five active galactic nuclei, one quasar (Craig \& Fruscione 1997), and another new magnetic CV, EUVE J2115-58.0 (previously reported; Craig 1996), bring the total number of identifications to 36 . The candi- 

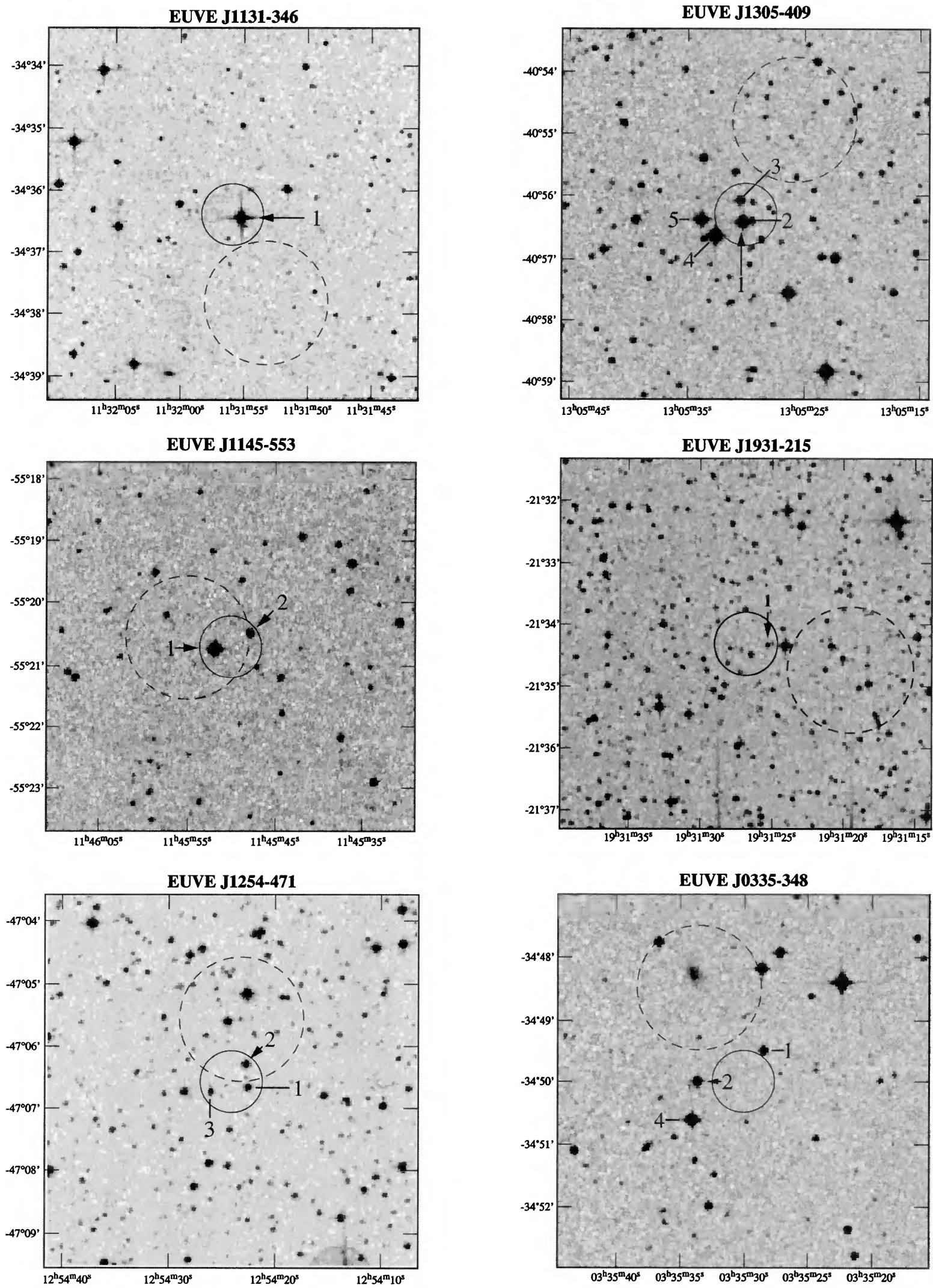

FIG. 1. (continued)

dates from the remaining fields observed showed no obvious optical signatures of EUV sources.

Table 2 presents a summary of the optical observation log and derived physical parameters for our sample of late-type and DA white dwarf counterpart candidates for the EUVE sources. Columns 1 through 10 give the EUVE source name, optical counterpart number as marked in the optical charts, right ascension and declination of the candidate as derived from the digitized Sky Survey maps, observation dates, exposure time, angular separation between the EUVE source 

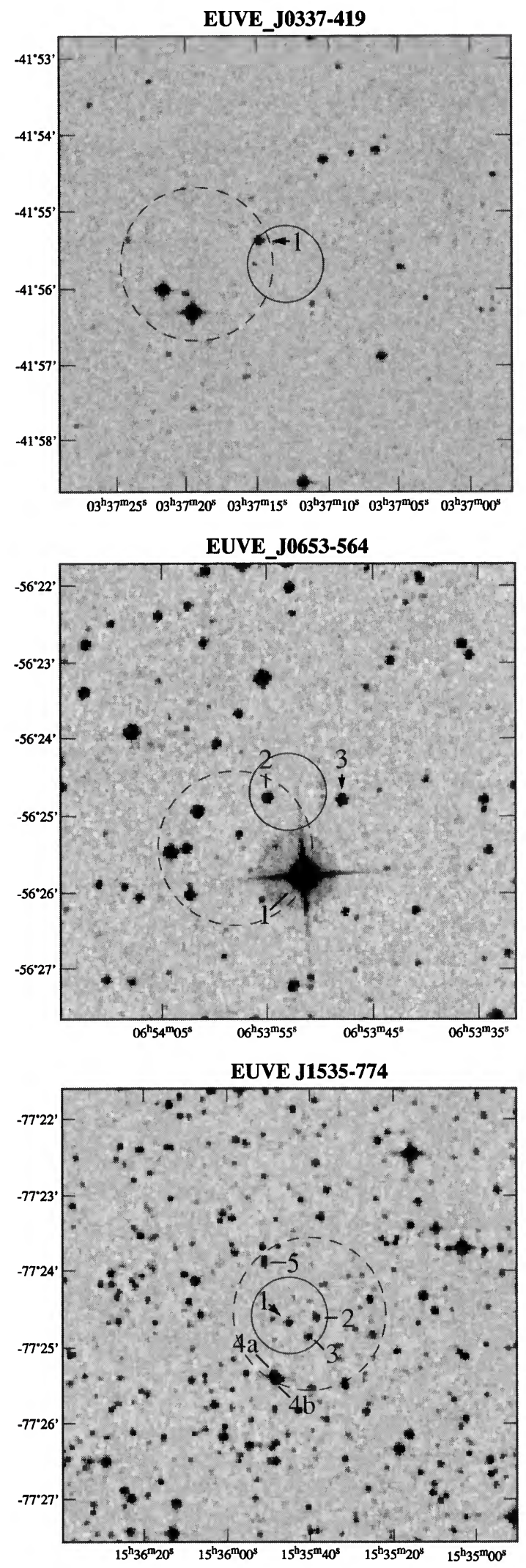

FIG. 1. (continued)

position and optical position of the candidate, angular separation between the optical and the ROSAT candidate, visual magnitude $m_{v}$, and the spectral type.

In Fig. 1 we show the finding charts for the fields studied. Possible source counterparts were first identified from the $6^{\prime} \times 6^{\prime}$ Digitized Sky Survey Maps. All positions are given in J2000 coordinates and charts centered at the ROSAT coordinates. The $30^{\prime \prime}$ radius positional error circle of ROSAT is shown on the chart as solid circle. The positions of EUV sources are determined from the EUVE data, and they are accurate to $1^{\prime}$ at the $90 \%$ confidence level and are shown as dashed circles. We observed marked candidates in each field and derived spectral types for all observed candidates. The EUVE optical counterpart is marked with an asterisk in column 2 of Table 2.

\section{IDENTIFICATION PROCEDURES AND RESULTS}

We compare the observed spectra with the spectral atlas of Jacoby et al. (1984) and determine the spectral types to within three subclasses. The empirical relation of spectral type and the depression of the $\mathrm{TiO}$ band (4760 $\AA$ ) relative to the continuum from Mathioudakis (1992) is also applied as a check to verify the spectral types obtained with the spectral atlas method. In the cases where the optical candidate is chosen as the EUV counterpart, the atlas of Pettersen \& Hawley (1989) was used for further detailed comparison. The visual magnitude, $m_{v}$, is derived by folding the measured absolute flux from the optical spectra of the $V$ band (5500 $\AA$ ) having a flux of a star with visual magnitude $m_{v}=0$, using absolute calibration photometry of Bessel (1979). Absolute visual magnitudes were obtained using the empirical correlation between the strength of the absorption band of $\mathrm{TiO} 4760 \AA$ and the absolute magnitude calibrated by Pettersen \& Hawley (1989).

Once the absolute magnitude is obtained, the spectroscopic distance can be estimated from the distance modulus. For the EUV candidates we estimated the neutral hydrogen column $N(\mathrm{H})$ density along the line of sight to verify the distances using a three-dimensional interpolation method of P. Jelinsky (1996, private communication). This model uses a large database of hydrogen column densities (Fruscione et al. 1994) from $E U V E$ and $N_{\mathrm{H}}$ measurements for individual stars. Generally, derived interstellar column densities are consistent with the observed EUV count rates. There are five sources for which the estimated columns are high, although derived distances are still reasonable. Higher columns could be a consequence of the $N(\mathrm{H})$ modeling, which in some directions is particularly affected by the presence of only a few direct measurements in the database.

\subsection{The $K$ Stars and M Stars}

More than $70 \%$ of the fields identified in this sample showed the characteristic spectral emission signature of $\mathrm{dK}$ or dM stars. Five of these 22 late-type stars are mid-to-late dKe types. These $\mathrm{dK}$ types show the telltale metallic lines, e.g., strong $\mathrm{Ca} \mathrm{H}$ and $\mathrm{K}$ lines and molecular bands such as TiO at $4760 \AA$, for later types. The summary of Balmer line fluxes, equivalent widths, spectral types, distances, and the estimated column densities for sources chosen as the optical counter part to the EUV source are presented in Table 3. Figure 2 shows a sample optical spectrum of EUVE J0426 -379 . 
TABLE 3. Results for EUV optical counterparts.

\begin{tabular}{|c|c|c|c|c|c|c|c|c|c|c|c|c|c|c|c|}
\hline \multirow[b]{3}{*}{$\begin{array}{l}\text { EUVE Name } \\
\text { (1) }\end{array}$} & \multicolumn{5}{|c|}{ Line Fluxes } & \multicolumn{5}{|c|}{ Equivalent Widths } & \multirow{3}{*}{$\begin{array}{c}\mathrm{TiO} \\
\text { band } \\
(12)\end{array}$} & \multirow{3}{*}{$\begin{array}{l}\text { Sp. } \\
\text { Type } \\
(13)\end{array}$} & \multirow[b]{3}{*}{$\begin{array}{c}d \\
(\mathrm{pc}) \\
(14)\end{array}$} & \multirow{3}{*}{$\begin{array}{c}\log N_{\mathrm{H}} \\
\left(\mathrm{cm}^{-2}\right) \\
(15)\end{array}$} & \multirow{3}{*}{$\begin{array}{l}\text { Obs. } \\
\text { Flux }^{\mathrm{a}} \\
(16)\end{array}$} \\
\hline & $\mathrm{Ca} \mathrm{K}$ & $\mathrm{Ca} \mathrm{H}$ & $\mathrm{H} \alpha$ & $\mathrm{H} \beta$ & $\mathrm{H} \gamma$ & \multirow{2}{*}{$\begin{array}{c}\mathrm{Ca} \mathrm{K} \\
(\AA) \\
(7)\end{array}$} & \multirow{2}{*}{$\begin{array}{c}\mathrm{Ca} \mathrm{H} \\
(\AA) \\
(8)\end{array}$} & \multirow{2}{*}{$\begin{array}{l}\mathrm{H} \alpha \\
(\AA) \\
(9)\end{array}$} & \multirow{2}{*}{$\begin{array}{c}\mathrm{H} \beta \\
(\AA) \\
(10)\end{array}$} & \multirow{2}{*}{$\begin{array}{c}\mathrm{H} \gamma \\
(\AA) \\
(11)\end{array}$} & & & & & \\
\hline & (2) & $\begin{array}{c}\left(10^{-14}\right. \\
(3)\end{array}$ & $\mathrm{cm}^{-2} \mathrm{~s}$ & $\begin{array}{c}\left.\AA^{-1}\right) \\
(5)\end{array}$ & (6) & & & & & & & & & & \\
\hline EUVE J0106-63 & 4.9 & 5.9 & 19.2 & 6.6 & 6.6 & 13.9 & 13.2 & 4.8 & 5.3 & 10.2 & 0.68 & $\mathrm{dM} 4 \mathrm{e}$ & 24 & 17.80 & 3.5 \\
\hline EUVE J0239-196 & 5.6 & 3.7 & 10.6 & 3.9 & 0.4 & 5.4 & 2.8 & 1.7 & 0.9 & 0.12 & 0.83 & $\mathrm{dM} 0 \mathrm{e}$ & 46 & 18.58 & 0.82 \\
\hline EUVE J0254-511 & 7.8 & 8.2 & 25.5 & 9.0 & 9.5 & 10.5 & 8.7 & 2.7 & 2.7 & 4.8 & 0.74 & $\mathrm{dM} 3 \mathrm{e}$ & 14 & 18.88 & 7.0 \\
\hline EUVE J0318-363 & 9.1 & 9.4 & 34.2 & 12.9 & 11.7 & 11.2 & 8.7 & 2.8 & 3.3 & 5.6 & 0.72 & $\mathrm{dM} 4 \mathrm{e}$ & 10 & 17.92 & 5.5 \\
\hline EUVE J0352-492 & 0.7 & 0.3 & 5.7 & $\ldots$ & $\cdots$ & 1.5 & 0.6 & 2.5 & $\cdots$ & $\cdots$ & 0.97 & $\mathrm{dK} 5 \mathrm{e}$ & 132 & 19.10 & 0.80 \\
\hline EUVE J0426-379 & 12.3 & 12.1 & 36.7 & 19.0 & 4.6 & 3.0 & 2.4 & 1.9 & 1.2 & 4.0 & 0.91 & $\mathrm{dK} 4 \mathrm{e}$ & 40 & 17.11 & 7.0 \\
\hline EUVE J0450-086 & 0.4 & 6.7 & 0.6 & 1.6 & 2.0 & 0.5 & 5.1 & 0.2 & 0.5 & 0.9 & 0.93 & $\mathrm{dK} 4 \mathrm{e}$ & 87 & 19.47 & 21.0 \\
\hline EUVE J0609-358 & 37.0 & 37.5 & 71.3 & 35.9 & 33.0 & 11.4 & 9.2 & 2.7 & 3.0 & 4.3 & 0.74 & $\mathrm{dM} 4 \mathrm{e}$ & 8 & 18.30 & 5.0 \\
\hline EUVE J0625-600 & 18.9 & 22.5 & 63.6 & 23.9 & 19.0 & 22.0 & 20.0 & 6.2 & 7.7 & 11.6 & 0.67 & dM5e & 10 & 18.50 & 2.1 \\
\hline EUVE J0702-018 & 1.4 & 1.3 & 4.0 & 1.9 & 1.3 & 15.6 & 10.0 & 3.3 & 4.3 & 5.2 & 0.76 & $\mathrm{dM} 2 \mathrm{e}$ & 72 & $<19.28$ & 15.0 \\
\hline EUVE J0749-766 & 29.4 & 32.0 & 106.0 & 45.2 & 36.9 & 14.4 & 11.7 & 4.2 & 5.4 & 8.2 & 0.68 & dM5e & 6 & 18.55 & 10.2 \\
\hline EUVE J0817-822 & 16.7 & 21.7 & 105.0 & 33.2 & 22.9 & 22.0 & 21.3 & 6.6 & 7.8 & 10.9 & 0.67 & dM5e & 8 & 18.20 & 5.0 \\
\hline EUVE J0825-163 & 22.0 & 15.8 & 40.0 & 12.3 & 5.0 & 4.6 & 2.6 & 1.0 & 0.5 & 0.5 & 0.89 & $\mathrm{dM} 0 \mathrm{e}$ & 18 & 18.77 & 8.0 \\
\hline EUVE J0835-430 & 0.04 & 0.03 & 0.01 & 0.01 & $\cdots$ & 9.0 & 5.0 & 1.6 & 0.4 & $\cdots$ & 0.91 & M1e & 138 & 20.80 & $1360.0^{\mathrm{b}}$ \\
\hline EUVE J0836-449 & 0.05 & 0.01 & 0.01 & 0.05 & 0.01 & 2.3 & 4.0 & 0.2 & 4.2 & 0.5 & 0.81 & M1e & 132 & 19.60 & 20.5 \\
\hline EUVE J1118-569 & 1.5 & 1.9 & 3.6 & 1.8 & 1.7 & 11.1 & 11.7 & 2.2 & 2.8 & 4.5 & 0.75 & $\mathrm{dM} 3 \mathrm{e}$ & 33 & 19.30 & 8.4 \\
\hline EUVE J1131-346 & 36.7 & 44.1 & 198.0 & 55.5 & 36.5 & 32.5 & 30.7 & 15.1 & 12.2 & 13.8 & 0.72 & $\mathrm{dM} 2 \mathrm{e}$ & 24 & 17.70 & 5.7 \\
\hline EUVE J1145-553 (1) & 13.5 & 11.9 & 37.8 & 13.7 & $\cdots$ & 1.4 & 1.1 & 1.0 & 0.4 & $\ldots$ & 0.88 & $\mathrm{dK} 5 \mathrm{e}$ & 32 & 18.90 & 6.0 \\
\hline EUVE J1145-553 (2) & 12.3 & 15.0 & 37.0 & 14.5 & 11.9 & 26.8 & 33.8 & 7.9 & 9.3 & 12.5 & 0.64 & $\mathrm{dM} 3 \mathrm{e}$ & 13 & 18.60 & 5.0 \\
\hline EUVE J1254-471 & 3.1 & 5.2 & 13.9 & 7.1 & 5.2 & 27.0 & 41.9 & 8.6 & 15.9 & 24.0 & 0.69 & $\mathrm{dM} 3 \mathrm{e}$ & 38 & 19.10 & 11.4 \\
\hline EUVE J1305-409 & 2.4 & 2.7 & 7.9 & 2.8 & 0.2 & 4.9 & 4.4 & 2.0 & 1.1 & 0.1 & 0.85 & dM1e & 14 & 18.80 & 8.4 \\
\hline EUVE J1931-215 & 17.0 & 20.3 & $\cdots$ & 19.7 & 15.0 & 16.0 & 16.7 & $\cdots$ & 6.6 & 7.5 & 0.70 & dM5e & 10 & $>18.90$ & 7.7 \\
\hline
\end{tabular}

${ }^{\mathrm{a}}$ The observed flux derived from EUVE Lexan count rates. Units are $10^{-13} \mathrm{erg} \mathrm{cm}^{-2} \mathrm{~s}^{-1}$.

${ }^{b}$ The observed flux derived using $N_{\mathrm{H}}$ value of $7.0 \times 10^{20} \mathrm{~cm}^{-2}$ (see Sec. 4.1).

The $\mathrm{M}$ stars were easily recognizable by their strong TiO bands. Emission from the Balmer series and $\mathrm{Ca} \mathrm{H}$ and $\mathrm{K}$ lines is notable. Sample spectra for dMe subclasses 0 to 5 are shown in Figs. 3(a)-3(f), respectively. Subclasses for the dMe's, which were obtained by using the empirical relation of the TiO band at $4760 \AA$ to the continuum, generally agreed with types chosen from atlases. Deeper depression of the TiO band corresponds to later M stars, but we classify a few stars with intermediate values as later $M$ types. For example, EUVE J0609-358 had a TiO ratio of 0.74 but is classified as a dM4e. This particular star had very strong emission, which may slightly bias determination of the continuum. A second possible source of uncertainty comes from the fact that the $\mathrm{MgH}$ band at $4780 \AA$ is stronger for later types and is not always resolved for our moderate resolution survey.

We have calculated observed fluxes for our sample (see Mathioudakis et al. 1995b) using the EUVE Lexan count rates, the coronal model of Monsignori Fossi \& Landini (1994) with $\log T=6.7$, and the derived column densities mentioned above. Although dependence of the fluxes on column density is small ( $50 \%$ changes in $N_{\mathrm{H}}$ result in flux changes of $\leqslant 25 \%$ ), several sources have derived columns near the limit for sources detectable in the Lexan bandpass. Luminosities for such sources then exceed those expected from coronal emission of late-type stars. For such sources we have used a maximum canonical value of $N_{\mathrm{H}}$ of $7.0 \times 10^{20}$, as is noted in Table 3. Most of the the large $N_{\mathrm{H}}$ values result from not having enough sources in the three-dimensional interpolation to give an accurate estimate of column. EUVE J0835-430 and EUVE J0836-449 are the exception, and their reddened spectra support their large derived column densities (discussed below).

Several sources have other notable characteristics. We discuss these individual cases presently.

EUVE J0318-363 can be associated with the 13th magnitude proper-motion star, BPM 48175, which suggests EUV and soft x-ray surveys are sensitive to fainter nearby dwarf stars and, perhaps, an uncataloged population.

EUVE J0426-379 is within $1^{\prime}$ of the ROSAT source 1RXS J042616.9-375730 and is also positionally coincident with PPM 28022 (CD - 38 1575), a 10th magnitude high proper-motion star. In addition, a very low column density

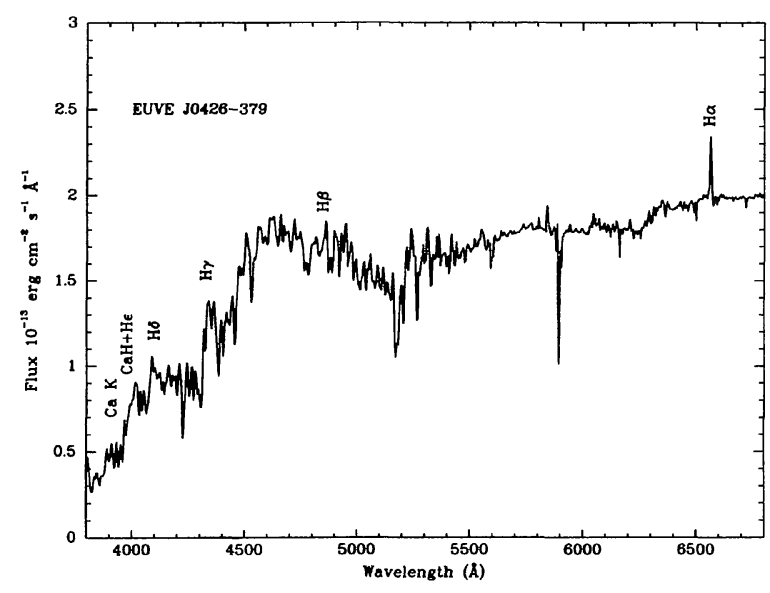

FIG. 2. Sample optical spectrum of dK4e star, EUVE J0426-379. 

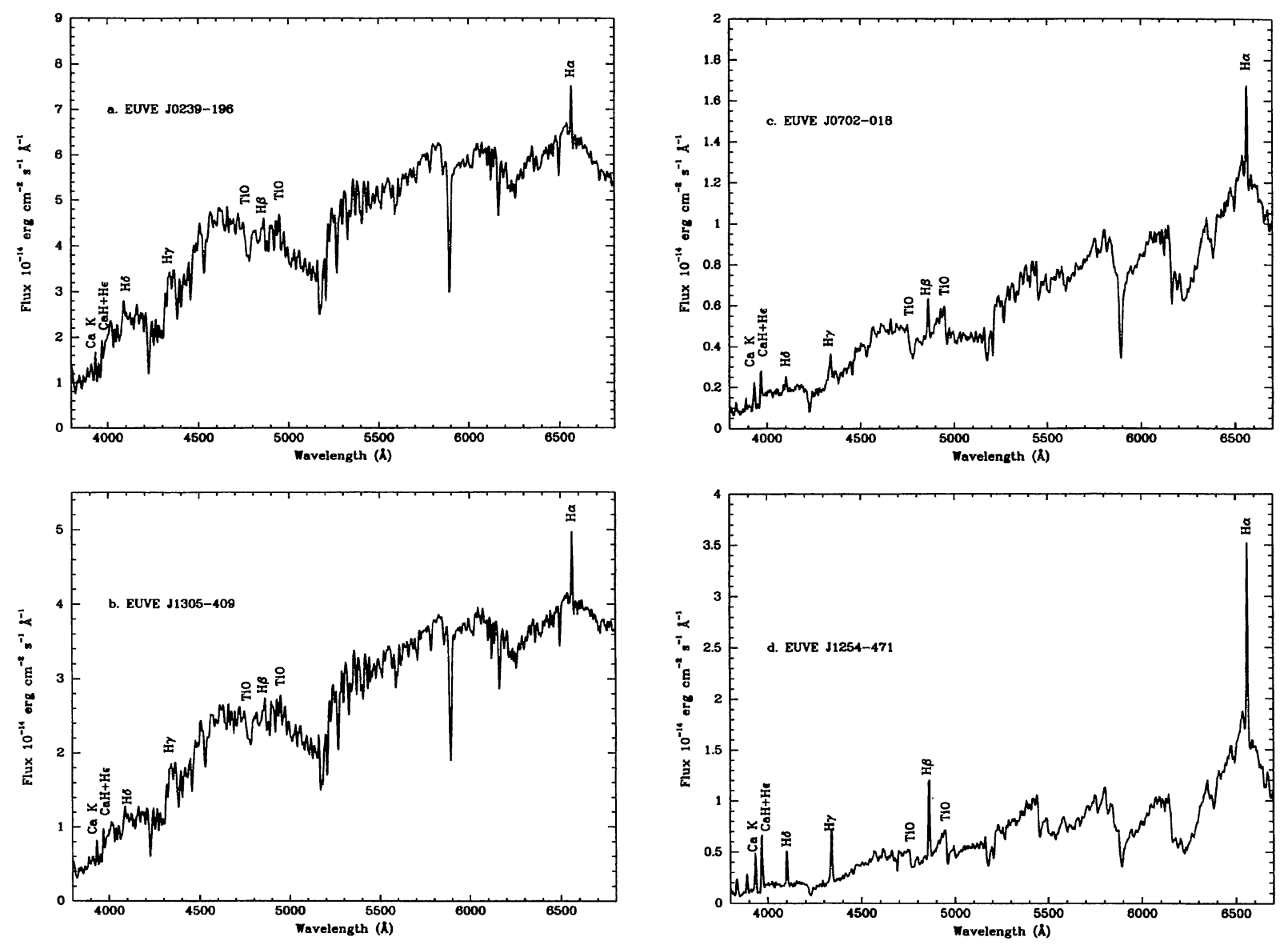

FIG. 3. Sample optical spectra of the Me stars.

$\left(\log N_{\mathrm{H}}=17.1\right)$ in the direction of EUVE J0426-379 is consistent with this source's marginal detection in the Al/C (200 $\AA$ ) band of 0.026 counts s ${ }^{-1}$.

EUVE J0352-492 has a similarly moderate column density of $\log N_{\mathrm{H}}=20.3$, which is consistent with its $\mathrm{Al} / \mathrm{C}$ detection of 0.003 counts $\mathrm{s}^{-1}$ in the EUVE RAP (McDonald et al. 1994).

EUVE J0625-600 is coincident with Einstein slew source 1ES 0625-60 (Elvis et al. 1992).

EUVE J0749-766 changed from a NOID to a dMe identification in the Bowyer et al. (1996) second EUVE catalog based on preliminary optical identification work. Our finding of a dM5e spectral type is a refinement of this change.

EUVE J0835-430 and EUVE J0836-449 are two of the faintest sources identified in this study $\left(m_{v} \sim 18\right)$. Although Balmer series and $\mathrm{Ca} \mathrm{H}$ and $\mathrm{K}$ emission is easily discernible in their spectra, their luminosity class is not. However, to obtain a reasonable distance determination and column density they must be main-sequence stars. The columns derived from the $N_{\mathrm{H}}$ database are at the upper limit for sources detectable by $E U V E$. This fact is supported by the large amount of reddening seen in their spectra, which is in agreement with the reddening given by Neckel \& Klare (1980), $1.9 \leqslant A_{V} \leqslant 2.6$, for their Galactic positions.

EUVE J1131-346 shows very strong emission, e.g., $\mathrm{H}$ $\alpha$ is a factor of 20 to 50 stronger than the other dM1-2e's in this survey (see Fig. 4). EUVE J1131-346 is coincident with the possible isolated T Tauri star PDS 55, which was discussed in an IRAS survey by Gregorio-Hetem et al. (1992). The strong emission lines detected here support the idea that this source is an isolated $\mathrm{T}$ Tauri star, but higher resolution follow-up spectroscopy is needed for confirmation.

EUVE J1145-553 has two probable candidates, dK5e and $\mathrm{dM} 3 \mathrm{e}$ stars. The $\mathrm{dKe}$ source is the more likely EUVE source since it is only $33^{\prime}$ from the EUVE center, although we cannot rule out the contribution from the dM3e. EUVE J1145-553 is coincident with Einstein slew source 1ES 1143-55.0 (Elvis et al. 1992).

EUVE J1931-215 was identified in the second EUVE catalog (Bowyer et al. 1996) as a dMe based on preliminary results of this work. We now refine that identification as a dM5e.

\section{WHITE DWARFS}

We present four DA white dwarfs discovered as possible optical counterparts of faint EUVE/ROSAT non-identified sources. The optical spectra of these white dwarfs are shown in Figs. 5(a)-5(d).

We determine the atmospheric parameters by fitting the Balmer line profiles with the theoretical line profiles of 

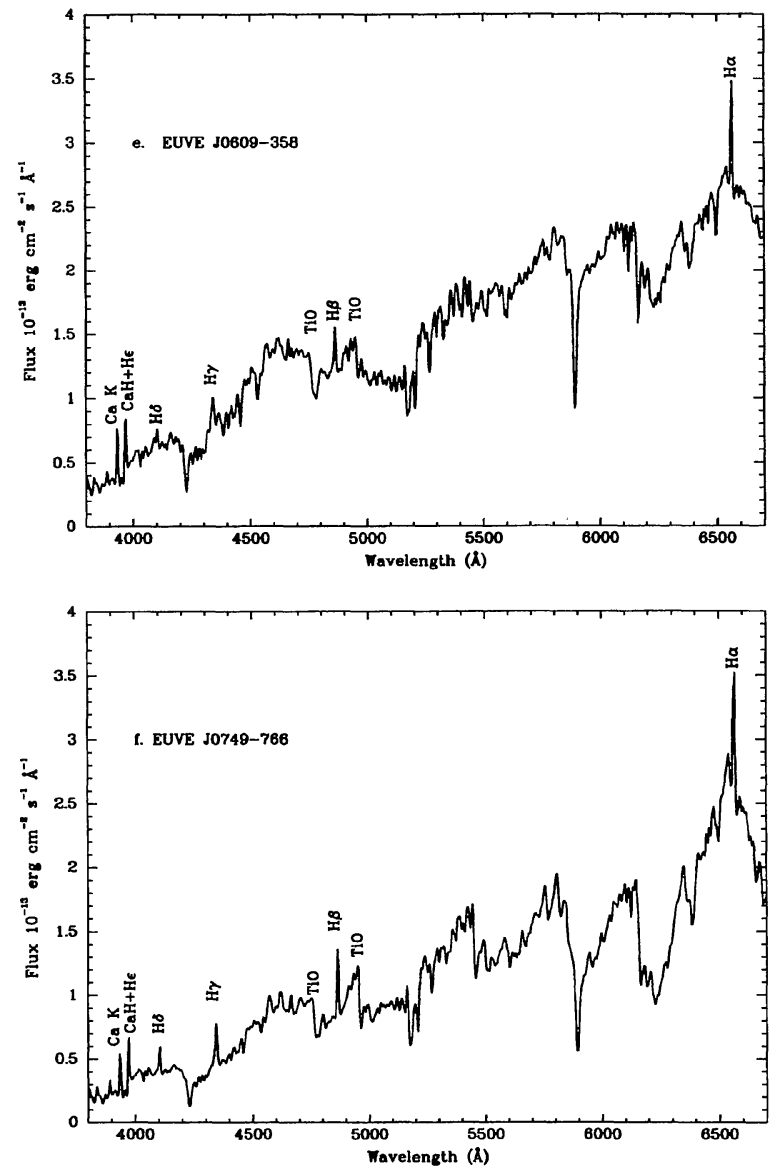

FIG. 3. (continued)

Wesemael et al. (1980). We use the profiles computed with the assumption of local thermodynamic equilibrium, pure hydrogen composition, and hydrogen line blanketing. We fit a combination of the $\mathrm{H} \alpha, \mathrm{H} \beta, \mathrm{H} \gamma$, and $\mathrm{H} \epsilon$ lines. In some cases, we had to exclude either or both $\mathrm{H} \alpha$ and $\mathrm{H} \epsilon$ lines because of a large uncertainty about the placement of the continuum. Table 4 lists the parameters derived for each white dwarf, and Fig. 6 shows the best fits to the Balmer lines. The four white dwarfs are the likely optical counterparts considering their high effective temperatures. We also estimate the absolute magnitudes using mass-radius relations derived from the Wood (1995) cooling sequence for a carbon white dwarf surrounded by $\mathrm{He}$ and $\mathrm{H}$ envelopes of $10^{-2}$ and $10^{-4}$ fractional mass and from the model atmospheres fluxes of Wesemael et al. (1980). Photometric distances are then estimated in combination with the visual magnitudes listed in Table 2.

We have also analyzed the EUVE Lexan filter count rates in order to constrain the interstellar column densities of hydrogen. The EUV fluxes of the white dwarfs are computed using synthetic spectra from a grid of LTE pure hydrogen models calculated using the code of Koester (1996). A table of synthetic count rates is calculated as a function of $T_{\text {eff }}$ and $N(\mathrm{H} \mathrm{I})$ for a fixed value of the gravity given by our Balmer line fits. The solid angles to the stars are fixed by normalizing the model spectra to their visual magnitudes. The abun-

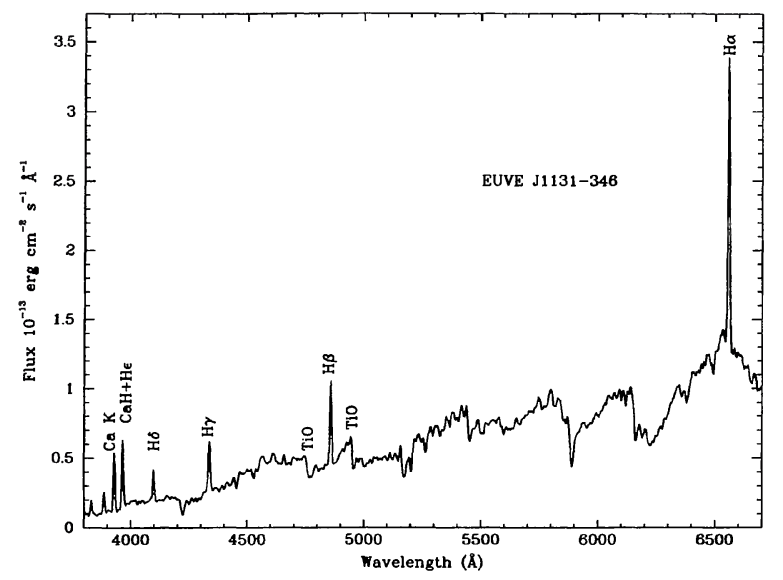

FIG. 4. Spectrum of EUVE J1131-346 showing strong Balmer emission.

dance ratios of interstellar $\mathrm{He} \mathrm{I}$ and $\mathrm{He}$ II to $\mathrm{H} \mathrm{I}$ are, respectively, fixed to 0.07 and 0.03 . These ratios are representative of values measured in other lines of sight (e.g., Dupuis et al. 1995 and Barstow et al. 1997). By interpolating in the grid, we determine the interval of $N(\mathrm{H} \mathrm{I})$ consistent with the range of optical $T_{\text {eff }}$ and the $1 \sigma$ error on the count rates. Table 4 summarizes our results.

The four white dwarfs fall in the range of effective temperature in which the abundances of heavy elements are generally low enough not to suppress the EUV emission (Barstow et al. 1993; Finley 1996; Wolff et al. 1996; Marsh et al. 1996a; Vennes et al. 1996). The joint detections by the ROSAT X-ray Telescope and in the EUVE Lexan band suggest the new white dwarfs have a low metal abundance. Unfortunately, with the limited broad-band, EUV/soft x-ray, photometric data we have, we cannot obtain more detailed information about their atmospheric composition. Because we do not know their true photospheric composition, the column densities we have measured are in reality upper limits on $N(\mathrm{H} \mathrm{I})$. We further comment on the individual stars:

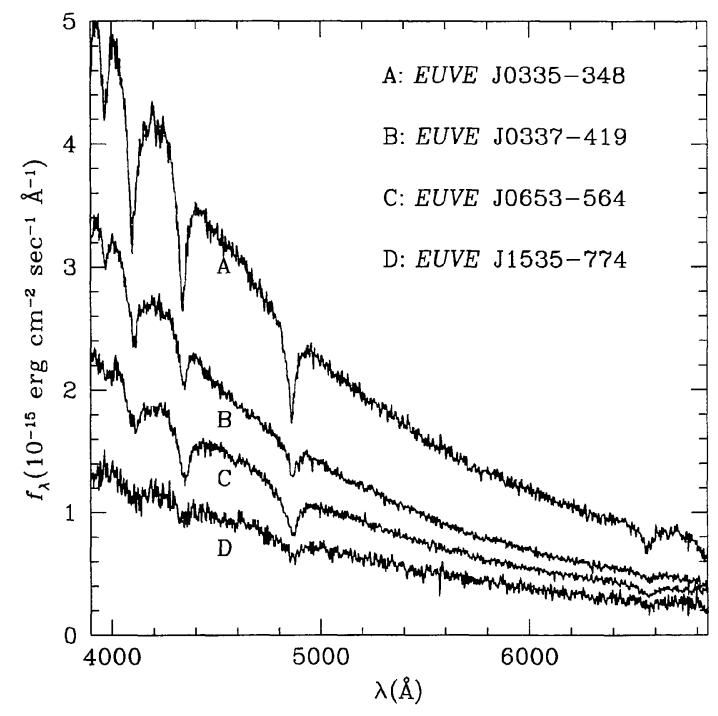

FIG. 5. Optical spectra of the white dwarfs. 
TABLE 4. Parameters for the hot white dwarfs.

\begin{tabular}{llccc}
\hline \hline EUVE Name & \multicolumn{1}{c}{$\begin{array}{c}\text { Distance } \\
(\mathrm{pc})\end{array}$} & $\begin{array}{c}N_{\mathrm{H} \mathrm{I}} \\
\left.\mathrm{em}^{-2}\right)\end{array}$ \\
\hline EUVE J0335-348 & $34848 \pm 600 \mathrm{~K}$ & $7.95 \pm 0.05$ & 187 & $2.83_{-0.61}^{+0.78}$ \\
EUVE J0337-419 & $51737 \pm 4000 \mathrm{~K}$ & $7.61 \pm 0.20$ & 398 & $1.08_{-0.11}^{+0.15}$ \\
EUVE J0653-564 & $37626 \pm 400 \mathrm{~K}$ & $8.59 \pm 0.15$ & 159 & $5.06_{-0.85}^{+1.12}$ \\
\hline \hline
\end{tabular}

EUVE J0335-348 has a surface gravity close to the average gravity of field white dwarfs. This source is typical of the brighter pure hydrogen white dwarfs detected in the EUVE all-sky survey.

EUVE J0337-419 is the hottest star in our sample and it has a rather low surface gravity. Above $50,000 \mathrm{~K}$, computations of equilibrium radiative levitation in DA envelopes by Chayer et al. (1995) predict a detectable amount of heavy elements in white dwarf atmospheres. The predicted abundances are sufficiently large to suppress the flux in the $E U V E$ Lexan band such that a star like EUVE J0337-419 should not be detected. The subsequent detection suggests that its abundances of heavy elements are lower than expected from the diffusion theory. EUVE J0337-419 would then be similar to the hot white dwarf $\mathrm{HZ} 43$, a $50,000 \mathrm{~K} \mathrm{DA}$ with a highly pure hydrogen atmosphere.

EUVE J0653-564, a high gravity star $(\log g=8.8)$, lies near the end of the surface gravity distribution of field white dwarfs measured by Bergeron et al. (1992). It therefore represents a rare occurrence of a white dwarf with a high mass. Vennes et al. (1997) have published an analysis of EUVE

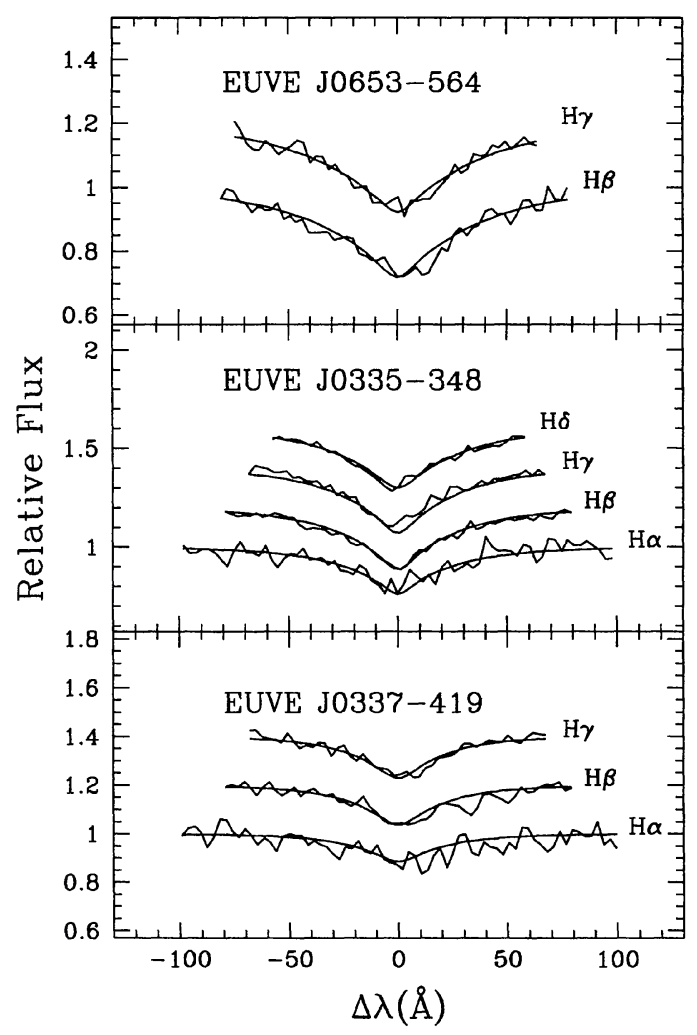

FIG. 6. (a)-(d) Best-fit solutions to Balmer lines (see Sec. 5).
J0653 - 564 based on different optical data and models and have obtained results in good agreement with ours.

EUVE J1535-774 is another high gravity star $(\log g$ in excess of 8.5), but our spectrum is noisy so this source is not included in Table 4. Vennes et al. (1997) have measured the parameters for this star based on a higher signal-to-noise ratio spectrum $\left(T_{\text {eff }}=58,000 \mathrm{~K}, \log g=9.14\right.$, and photometric distance of $107 \mathrm{pc})$. Assuming these parameters, we find a $N(\mathrm{H} \mathrm{I})$ of $9.1 \pm 2.5 \times 10^{19} \mathrm{~cm}^{-2}$. Vennes et al. discussed the detection of a larger number of massive white dwarfs
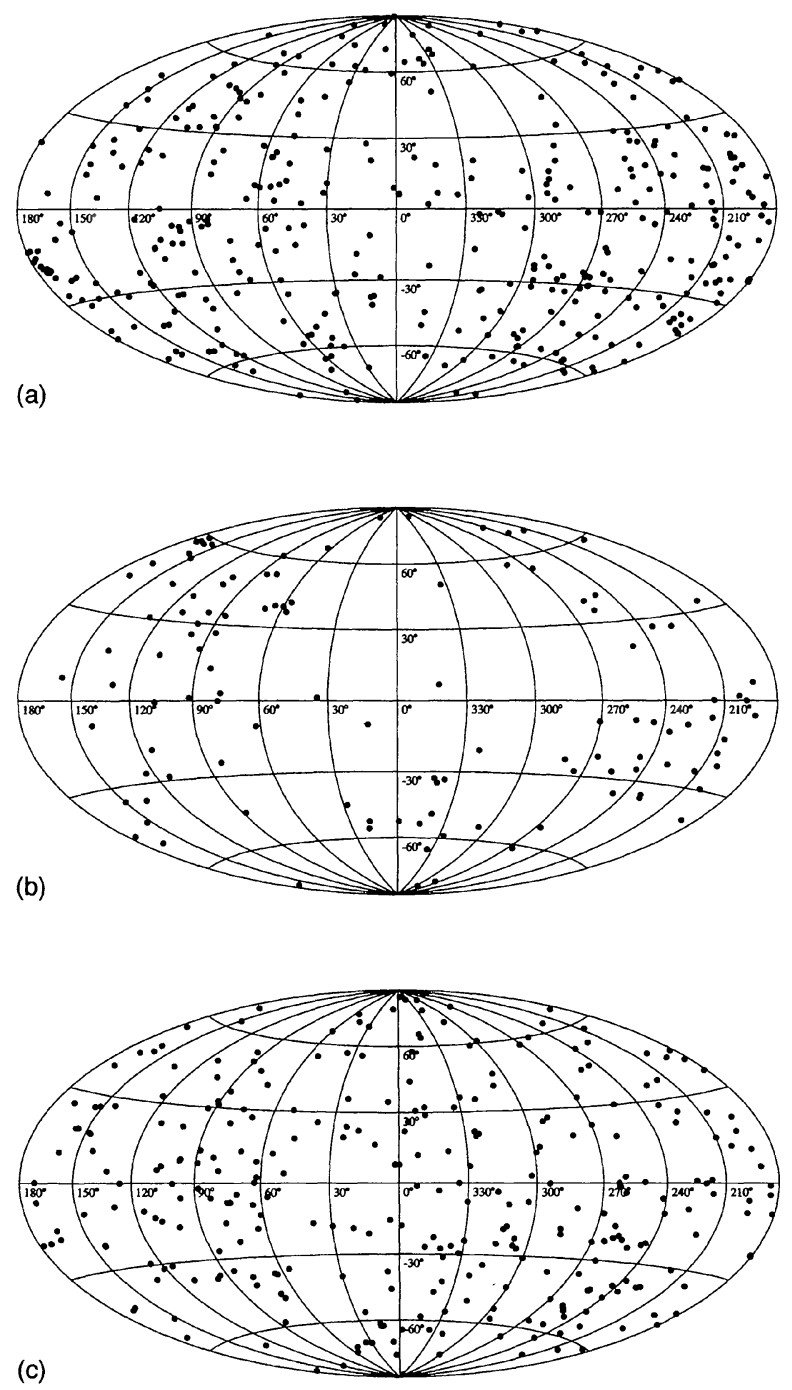

FIG. 7. Aitoff projection of $(a)$ late-type stars, $(b)$ white dwarfs, and (c) NOID sources in Galactic coordinates. 


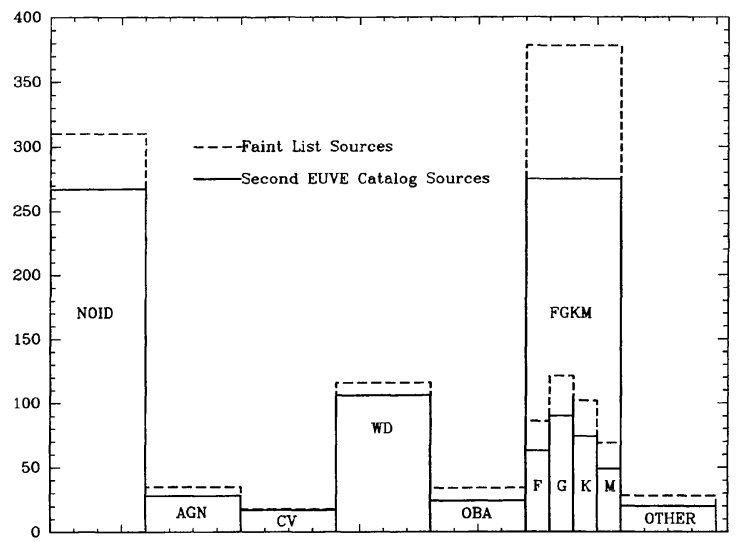

FIG. 8. The number distribution of 918 EUVE optical counterparts as a function of identification type (see Sec. 6).

among EUV-selected white dwarfs than in optical samples (e.g., Bergeron et al. 1992). Marsh et al. (1996b) reached a similar conclusion based on the ROSAT WFC sample. Thus, the detection of two massive white dwarfs out of four found in our optical identification campaign may not be a coincidence.

\section{EUV SOURCE DISTRIBUTION}

Before the EUV sky was fully mapped during the ROSAT WFC (Pye et al. 1995) and EUVE (Bowyer et al. 1996) surveys, a widespread perception held that interstellar opacity would limit the number of observable EUV sources to only a few. This theory now disproven, we show in Fig. 7 the distribution of the current late-type, white dwarf, and NOID sources in an Aitoff projection in Galactic coordinates ( $l=0^{\circ}$ at center and increasing leftward). We point out that distribution is a strong function of the local interstellar column density and that the spatial distribution of EUV sources is not isotropic. Diamond et al. (1995) and Welsh et al. (1994) reported the increased number of EUV sources in Galactic quadrant $3\left(180<l^{\Pi}<270, b^{\mathrm{I}}<0\right)$ where a particu- larly low $\mathrm{H}$ I column is known. We find $50 \%$ of our late-type stars, and 3 out of the 4 newly identified faint white dwarfs, are in the direction of quadrant 3 , further supporting this lower column direction enhancement. Lampton et al. (1997) also noted that the low number of faint objects in the direction of the 4th quadrant of the Galaxy, where nearby intervening $\mathrm{H}$ I column density is appreciable, also can be attributed to the non uniformity of the local ISM.

The source distribution of the combined Bowyer et al. (1996) and Lampton et al. (1997) catalogs is dominated by late-type stars $(\gtrsim 50 \%)$. The second largest class of identified objects are the white dwarfs $(\sim 21 \%)$ followed by earlytype stars, extragalactic objects, sources classified as "other," and cataclysmic variables (each contributing $\sim 10 \%$ ). In Fig. 8 we show the number distribution as a function of the 918 sources grouped by identification class. In this light, the fact that of $80 \%$ of the sources we are able to positively identify are late-type stars, followed by a handful of white dwarfs, is not surprising. Although we have presently only identified $\sim 10 \%$ of the EUVE unidentified objects, one is tempted to predict that the majority of these objects are active late-type stars, many of which may be high proper-motion objects and, generally, uncataloged.

We wish to thank the principal investigator of the EUVE project, and the EUVE science team for their advice and support. The authors would also like to thank M. Lampton for providing the faint source data before their publication, M. Mathioudakis for useful discussions, and D. Koester and D. Finley for providing grids of the DA synthetic spectra. We also thank the referee for the prompt report. This research has made use of data obtained through the High Energy Astrophysics Science Archive Research Center Online Service (HEASARC), provided by the NASA-Goddard Space Flight Center. The Digitized Sky Survey was produced at the Space Telescope Science Institute under US Government grant NAG W-2166. This work has been supported by NASA contracts NAS5-30180 and NAS5-29298. The Center for EUV Astrophysics is a division of UC Berkeley's Space Sciences Laboratory.

\section{REFERENCES}

Barstow, M. A., et al. 1993, MNRAS, 264, 16

Barstow, M. A., et al. 1997, MNRAS (in press)

Bergeron, P., Saffer, R. A., \& Liebert, J. 1992 ApJ, 394, 228

Bessel, M. S., 1979, PASP, 91, 589

Bowyer, S., \& Malina, R. F. 1991, in Extreme Ultraviolet Astronomy, edited

by R. F. Malina and S. Bowyer (Pergamon, New York), p. 391

Bowyer, S., Lieu, R., Lampton, M., Lewis, J., Wu, X., Drake, J. J., \&

Malina, R. F. 1994, ApJS, 93, 569

Bowyer, S., Lampton, M., Lewis, J., Wu, X., Jelinsky, P., \& Malina, R. F. 1996, ApJS, 102, 129

Chayer, P., Fontaine, G., \& Wesemael, F. 1995, ApJS, 99, 189

Craig, N., 1995 IAU Circ. No. 6201

Craig, N., 1996 IAU Circ. No. 6297

Craig, N., \& Fruscione, A. 1997, AJ, submitted

Craig, N., et al. 1995, AJ, 110, 1304

Diamond, C. J. , Jewell, S. J., \& Ponman, T. J. 1995, MNRAS, 274, 589

Dupuis, J., Vennes, S., Bowyer, S., Pradhan, A. K., \& Thejll, P. 1995, ApJ, 455,574
Elvis, M., Plummer, D., Schachter, J., \& Fabbiano, G. 1992, ApJS, 80, 257 Finley, D. S. 1996, in Astrophysics in the Extreme Ultraviolet, IAU Colloquium 152, edited by S. Bowyer and R. F. Malina (Kluwer, Dordrecht), p. 223

Fruscione, A., Hawkins, I., Jelinsky, P., \& Wiercigroch, A. 1994, ApJS 94, 127

Gregorio-Hetem, J., Lepine, J. R. D., Quast, G. R., Torres, C. A. O., \& De la Reza, R. 1992, AJ, 103, 549

Jacoby, G. H., Hunter, D. A., \& Christian, C. A. 1984, ApJS, 56, 257

Koester, D. 1996, in Astrophysics in the Extreme Ultraviolet, IAU Colloquium 152, edited by S. Bowyer and R. F. Malina (Kluwer, Dordrecht), p. 185

Lampton, M., Lieu, R., Schmitt, J. H. M. M., Bowyer, S., Voges, W., Lewis, J., \& Wu, X. 1997, ApJS, 108, 545

Marsh, M. C., et al. 1996a, MNRAS (in press)

Marsh, M. C., et al. 1996b, MNRAS (in press)

Mathioudakis, M. 1992, Ph.D. thesis, Queens University of Belfast

Mathioudakis, M., et al. 1995a, A\&A, 302, 422 
Mathioudakis, M., Fruscione, A., Drake, J. J., McDonald, K. Bowyer, S., \& Malina, R. F. 1995b, A\&A, 300, 775

McDonald, K., Craig, N., Sirk, M. M., Drake, J. J., Fruscione, A., Vallerga, J. V., \& Malina, R. F. 1994, AJ, 108, 1843

Monsignori-Fossi, B., \& Landini, M. 1994, Sol. Phys., 152, 81

Neckel, T., \& Klare, G. 1980, A\&AS, 42, 251

Pettersen, B., \& Hawley, S. L. 1989, A\&A, 217, 187

Pye, J. P., et al. 1995, MNRAS, 274, 1165

Vennes, S., Thejll, P. A., Genova, R., \& Dupuis, J. 1997, ApJ (in press)
Vennes, S., Thejll, P. A., Wickramasinghe, D. T., \& Bessell, M. S. 1996 ApJ, 467, 782

Voges, W., et al. 1997, A\&A (in press)

Welsh, B. Y., Craig, N., Vedder, P. W., \& Vallerga, J. V. 1994, ApJ, 437, 638

Wesemael, F., Auer, L. H., Van Horn, H. M., \& Savedoff, M. P. 1980, ApJS, 43, 159

Wolff, B., Jordan, S., \& Koester, D. 1996, A\&A, 307, 149

Wood, M. 1995, in Lecture Notes in Phys. 443, edited by K. Koester and Werner (Springer, Berlin), p. 41 